\title{
FORCED CUBIC SCHRÖDINGER EQUATION WITH ROBIN BOUNDARY DATA: CONTINUOUS DEPENDENCY RESULT
}

\author{
CHARLES BU ${ }^{1}$
}

(Received 30 May 1996)

\begin{abstract}
For the cubic Schrödinger equation $i u_{t}=u_{x x}+k|u|^{2} u, 0 \leq x, t<\infty$, initial data $u(x, 0)=u_{0}(x) \in H^{2}[0, \infty)$, and Robin boundary data $u_{x}(0, t)+\alpha u(0, t)=R(t) \in$ $C^{2}[0, \infty)$ (where $\alpha$ is real), we show that the solution $u$ depends continuously on $u_{0}$ and $R$.
\end{abstract}

\section{Preliminaries}

In recent years, a vast amount of work has been done on pure initial value problems for important nonlinear evolution equations such as NLS and KdV. No comparable attention has been given to mixed initial boundary-value problems for these equations. Yet in many cases of physical interest, the mathematical models lead precisely to problems where boundary data is non-zero (sometimes called "forced problems"). For example, the launching of solitary waves in a shallow water channel, and the excitation of ionacoustic solutions in a double plasma machine, belong to this class. In ionospheric modification experiments, one directs a radio frequency wave at the ionosphere. At the reflection point of the wave, a sufficient level of electron-plasma wave is excited to make the nonlinear behavior important [9]. This may be described by the cubic NLS equation with Dirichlet boundary conditions

$$
\begin{gathered}
i u_{t}=u_{x x} \pm 2|u|^{2} u, \quad 0 \leq x, \quad t<\infty \\
u(x, 0)=u_{0}(x), \quad u(0, t)=Q(t) .
\end{gathered}
$$

Under the assumption that $u_{0} \in H^{2}[0, \infty), Q \in C^{2}[0, \infty)$, global existence, wellposedness and blow-up of the solution to (1.1) were established in $[2,4,6]$. The

\footnotetext{
'Department of Mathematics, Wellesley College, Wellesley, MA 02482, USA

(C) Australian Mathematical Society 2000, Serial-fee code 0334-2700/00
} 
following forced nonlinear Schrödinger equation is also important:

$$
\begin{aligned}
i u_{t} & =u_{x x}+k|u|^{2} u, \quad 0 \leq x, \quad t<\infty, \\
u(x, 0) & =u_{0}(x), \quad u_{x}(0, t)+\alpha u(0, t)=R(t),
\end{aligned}
$$

where $\alpha$ and $k$ are real. This problem has significant physical implications. It arises from the propagation of optical solitons [5]. Also, it models water waves [1] when there is an additional term $u_{x}$ on the right-hand side and $\alpha \rightarrow \infty$. We notice that the boundary condition is of Robin type.

It was shown in [3] that for this equation, there exists a unique classical solution $u \in C^{0}\left(H^{2}\right) \cap C^{1}\left(L^{2}\right)$ provided that $u_{0} \in H^{2}[0, \infty), R \in C^{2}[0, \infty)$. In this article, we will show that the solution $u$ depends continuously on the initial and boundary data.

The following notation will be used throughout:

$$
\begin{gathered}
P(t)=u_{x}(0, t), \quad Q(t)=u(0, t), \quad R(0, t)=P(t)+\alpha Q(t), \\
\left\|u^{\prime}\right\|_{2}=\left[\int_{0}^{\infty}\left|u_{x}(x, t)\right|^{2} d x\right]^{1 / 2}, \\
\|u\|_{4}=\left[\int_{0}^{\infty}|u(x, t)|^{4} d x\right]^{1 / 4}, \\
\|u\|_{m, p}=\left[\int_{0}^{\infty} \sum_{|\alpha| \leq m}\left|D_{x}^{\alpha} u(x, t)\right|^{p} d x\right]^{1 / p}, \\
\|R\|_{C^{1}[0, T]}=\sup _{0 \leq t \leq T}\left[|R(t)|+\left|R^{\prime}(t)\right|\right] .
\end{gathered}
$$

In addition, we need the following Gagliardo-Nirenberg estimates [7] where $\lambda$ is a constant:

$$
\|u\|_{4}^{4} \leq \lambda\left\|u^{\prime}\right\|_{2}\|u\|_{2}^{3} .
$$

There are three interesting identities for (1.2):

$$
\begin{aligned}
\|u\|_{2}^{2}= & \left\|u_{0}\right\|_{2}^{2}-2 \Im\left(\int_{0}^{t} P(\tau) \overline{Q(\tau)} d \tau\right) \\
\left\|u^{\prime}\right\|_{2}^{2}-\frac{k}{2}\|u\|_{4}^{4}= & \left\|u^{\prime}\right\|_{2}^{2}-\frac{k}{2}\left\|u_{0}\right\|_{4}^{4}-2 \Re\left(\int_{0}^{t} P(\tau) \overline{Q^{\prime}(\tau)} d \tau\right) \\
\int_{0}^{\infty} u \bar{u}^{\prime} d x= & \int_{0}^{\infty} \bar{u}_{0} \bar{u}_{0}^{\prime} d x-\int_{0}^{t} Q(\tau) \overline{Q^{\prime}(\tau)} d \tau \\
& +i \int_{0}^{\infty}|P(\tau)|^{2} d \tau+\frac{k}{2} i \int_{0}^{t}|Q(\tau)|^{4} d \tau
\end{aligned}
$$


Based on these identities and Gagliardo-Nirenberg estimates, the following lemma can be derived directly from [3].

LEMMA 1. For any $T>0$, assume $R(t) \in C^{1}, u_{0} \in H^{1}$ and the initial-boundary data satisfy necessary compatibility conditions at $x=t=0$. If $\left\|u_{0}\right\|_{1,2}<M$, $\|R\|_{C^{l}[0, T]}<M$, then there exists $\tilde{\lambda}>0$ which only depends on $M, T$ such that $\|u\|_{1,2} \leq \tilde{\lambda}$ for $0 \leq t \leq T$ and $\|u\|_{\infty} \leq c\left\|u^{\prime}\right\|_{2}^{1 / 2}\|u\|_{2}^{1 / 2} \leq \lambda_{0}$.

We will consider (1.2) for $0 \leq t \leq T$ and $\left\|u_{0}\right\|_{1,2}<M(M, T<\infty$, arbitrary).

\section{Continuous dependency result}

Throughout this section we shall assume that $u, v$ solve (2.1) with data $\left(R, u_{0}\right)$ and $\left(R_{1}, v_{0}\right)$ lying in $C^{2}[0, T] \times H^{2}[0, \infty)=X$. According to the global existence theorem in [3], the map

$$
f: X \rightarrow Y=C^{1}\left(L^{2},[0, T]\right) \cap C^{0}\left(H^{2},[0, T]\right)
$$

via $\left(R, u_{0}\right) \rightarrow u$ is well-defined. To prove continuous dependency, we shall fix $z=\left(R, u_{0}\right) \in X$ and $z_{1}=\left(R_{1}, v_{0}\right) \in X$. Let $\|z\|_{X}=\max \left\{\|R\|_{C^{2}[0, T]},\left\|u_{0}\right\|_{2,2}\right\}<M$, $\left\|z_{1}\right\|_{X}<M$ and

$$
w=\Delta u=v-u, \quad \Delta z=z_{1}-z=\left(\Delta R, w_{0}\right)=\left(R_{1}-R, v_{0}-u_{0}\right) .
$$

Since $v=w+u$ solves the forced NLS (1.2), we have

$$
\begin{aligned}
i\left(w_{t}+u_{t}\right) & =w_{x x}+u_{x x}+k|w+u|^{2}(w+u) \\
& =w_{x x}+u_{x x}+k(w+u)^{2}(\bar{w}+\bar{u}) \\
& =w_{x x}+u_{x x}+k\left(|w|^{2} w+2 u|w|^{2}+u^{2} \bar{w}+2|u|^{2} w+\bar{u} w^{2}+|u|^{2} u\right) .
\end{aligned}
$$

But $u$ solves the forced NLS (1.2) as well, therefore $w$ solves the following initialboundary value problem:

$$
\begin{gathered}
i w_{t}=w_{x x}+k|w|^{2} w+2 k u|w|^{2}+k u^{2} \bar{w}+2 k|u|^{2} w+k \bar{u} w^{2}, \\
w_{x}(0, t)+\alpha w(0, t)=\Delta R, \quad w_{0}=v_{0}-u_{0} .
\end{gathered}
$$

We have the following lemma.

LEMMA 2. There exists $m>0$ such that $\sup _{0 \leq i \leq T}\|v-u\|_{2} \leq m\left\|z_{1}-z\right\|_{X_{0}}^{1 / 2}$ where $X_{0}=C[0, T] \times L^{2}[0, \infty)$. 
Proof. Write $\Delta R=R_{1}(t)-R(t), \Delta P=P_{1}(t)-P(t)=v_{x}(0, t)-u_{x}(0, t)$, $\Delta Q=Q_{1}(t)-Q(t)$. We calculate

$$
\begin{aligned}
i \partial_{t}|w|^{2}= & i w_{t} \bar{w}+i w \bar{w}_{t} \\
= & {\left[w_{x x}+k|w|^{2} w+2 k|w|^{2} u+k u^{2} \bar{w}+2 k w|u|^{2}+k w^{2} \bar{u}\right] \bar{w} } \\
& -w\left[\bar{w}_{x x}+k|w|^{2} \bar{w}+2 k|w|^{2} \bar{u}+k \bar{u}^{2} w+2 k \bar{w}|u|^{2}+k \bar{w}^{2} u\right] \\
= & w_{x x} \bar{w}-w \bar{w}_{x x}+2 k|w|^{2}(\bar{w} u-\bar{u} w)+k\left(u^{2} \bar{w}^{2}-\bar{u}^{2} w^{2}\right) \\
& +k|w|^{2}(\bar{u} w-u \bar{w}) \\
= & 2 i \Im\left(w_{x x} \bar{w}+2 k|w|^{2} \bar{w} u+k u^{2} \bar{w}^{2}+k|w|^{2} \bar{u} w\right) .
\end{aligned}
$$

Thus, by applying Lemma 1, we get

$$
\begin{aligned}
& \int_{0}^{\infty}|w|^{2} d x \\
& =\left\|w_{0}\right\|_{2}^{2}+2 \Im \int_{0}^{t}\left[\int_{0}^{\infty}\left(w_{x x} \bar{w}+2 k|w|^{2} \bar{w} u+k u^{2} \bar{w}^{2}+k|w|^{2} \bar{u} w\right) d x\right] d \tau \\
& =\left\|w_{0}\right\|_{2}^{2}-2 \Im \int_{0}^{t} \Delta P \Delta \bar{Q} d \tau \\
& \quad+2 \Im \int_{0}^{t} \int_{0}^{\infty}\left(2 k|w|^{2} \bar{w} u+k u^{2} \bar{w}^{2}+k|w|^{2} \bar{u}^{2} w\right) d x d \tau \\
& \leq\left\|w_{0}\right\|_{2}^{2}-2 \Im \int_{0}^{t}(\Delta R-\alpha \Delta Q) \Delta \bar{Q} d \tau \\
& \quad+2|k| \int_{0}^{t} \int_{0}^{\infty}\left(3|w|^{3}|u|+|u|^{2}|w|^{2}\right) d x d \tau \\
& \leq\left\|w_{0}\right\|_{2}^{2}+2\left(\int_{0}^{t}|\Delta R(\tau)|^{2} d \tau\right)^{1 / 2}\left(\int_{0}^{t}|\Delta Q(\tau)|^{2} d \tau\right)^{1 / 2} \\
& \quad+2|k| \int_{0}^{t} \int_{0}^{\infty}\left(3|w|^{3}|u|+|u|^{2}|w|^{2}\right) d x d \tau
\end{aligned}
$$

Direct calculation shows

$$
\begin{aligned}
|Q(t)|^{2} & =|Q(0)|^{2}+\Re \int_{0}^{\infty} u_{0} \bar{u}_{0}^{\prime} d x-\Re \int_{0}^{\infty} u \bar{u}^{\prime} d x \\
& =\left|u_{0}(0)\right|^{2}+\left\|u_{0}^{\prime}\right\|_{2}\left\|u_{0}\right\|_{2}-\Re \int_{0}^{\infty} u \bar{u}^{\prime} d x \\
& \leq c_{0}+\|u\|_{2}\left\|u^{\prime}\right\|_{2} \\
& \leq c_{0}+\tilde{\lambda}^{2},
\end{aligned}
$$


and, from Lemma 1, we also get the following

$$
\begin{aligned}
\left|Q_{1}(t)\right|^{2} & =\left|Q_{1}(0)\right|^{2}+\Re \int_{0}^{\infty} v_{0} \bar{v}_{0}^{\prime} d x-\Re \int_{0}^{\infty} v \bar{v}^{\prime} d x \\
& =\left|v_{0}(0)\right|^{2}+\left\|v_{0}^{\prime}\right\|_{2}\left\|v_{0}\right\|_{2}-\mathfrak{R} \int_{0}^{\infty} v \bar{v}^{\prime} d x \\
& \leq c_{0}^{\prime}+\|v\|_{2}\left\|v^{\prime}\right\|_{2} \\
& \leq c_{0}^{\prime}+\tilde{\lambda}^{2} .
\end{aligned}
$$

Thus

$$
\begin{aligned}
\left(\int_{0}^{t}|\Delta Q(\tau)|^{2} d \tau\right)^{1 / 2} & \leq\left[\int_{0}^{t}\left(2\left|Q_{1}(\tau)\right|^{2}+2|Q(\tau)|^{2}\right) d \tau\right]^{1 / 2} \\
& \leq\left(2 T\left(c_{0}+\tilde{\lambda}^{2}\right)+2 T\left(c_{0}^{\prime}+\tilde{\lambda}^{2}\right)\right)^{1 / 2} \\
& =m_{0} .
\end{aligned}
$$

Substitute (2.10) in (2.7):

$$
\int_{0}^{\infty}|w|^{2} d x \leq\left\|w_{0}\right\|_{2}^{2}+\hat{c}\|\Delta R\|_{C[0, T]}+\tilde{c} \int_{0}^{t} \int_{0}^{\infty}|w|^{2} d x d \tau .
$$

Apply the Gronwall lemma to (2.11):

$$
\begin{aligned}
\int_{0}^{\infty}|w|^{2} d x & \leq\left(\left\|w_{0}\right\|_{2}^{2}+\hat{c}\|\Delta R\|_{C[0, T]}\right) e^{\tilde{c} t} \\
& \leq\left(\left\|w_{0}\right\|_{2}^{2}+\hat{c}\|\Delta R\|_{C[0, \eta]}\right) e^{i \bar{c} T}
\end{aligned}
$$

Therefore,

$$
\begin{aligned}
\sup _{0 \leq t \leq T}\left(\int_{0}^{\infty}|w|^{2} d x\right)^{1 / 2} & \leq \sqrt{\left(\left\|w_{0}\right\|_{2}^{2}+\hat{c}\|\Delta R\|_{C[0, T]}\right) e^{c T}} \\
& \leq m_{0}\left(\left\|w_{0}\right\|_{2}+c\|\Delta R\|_{C[0, T]}^{1 / 2}\right) \\
& =m_{0}\left(\left\|w_{0}\right\|_{2}^{1 / 2}\left\|w_{0}\right\|_{2}^{1 / 2}+c\|\Delta R\|_{C[0, T]}^{1 / 2}\right) \\
& \leq m_{0}\left(\sqrt{\left\|u_{0}\right\|_{2}+\left\|v_{0}\right\|_{2}}\left\|w_{0}\right\|_{2}^{1 / 2}+c\|\Delta R\|_{C[0, T]}^{1 / 2}\right) \\
& \leq m_{0}\left(\sqrt{2 R}\left\|w_{0}\right\|_{2}^{1 / 2}+c\|\Delta R\|_{C[0, T]}^{1 / 2}\right) \\
& \leq \max \left\{m\left\|w_{0}\right\|_{2}^{1 / 2}, m\|\Delta R\|_{C[0, T]}^{1 / 2}\right\} \\
& =m\|\Delta z\|_{X_{0}}^{1 / 2} \\
& =m\left\|z_{1}-z\right\|_{X_{0}}^{1 / 2} .
\end{aligned}
$$

This proves the lemma. 
LEMMA 3. There exists $\tilde{c}>0$ such that $\|v-u\|_{Y_{2}} \leq \tilde{c}\left\|z_{1}-z\right\|_{X}^{1 / 2}$ where $Y_{2}=$ $C^{1}\left(L^{2},[0, T]\right)$.

PROOF. The norm of $u$ on $Y_{2}$ is $\sup _{0 \leq 1 \leq T}\left(\left\|u_{t}\right\|_{2}+\|u\|_{2}\right)$. Since $\sup _{0 \leq t \leq T}\|u\|_{2,2}<\infty$, it is clear that RHS of (2.4) satisfies the local-Lipschitz condition on $w$ in $H^{2}$. Similar to the technique in [3], we show that $w=v-u$ is the unique global solution satisfying (2.4).

For $\alpha$ real, $\Delta R \in C^{2}[0, \infty), u \in H^{2}[0, \infty)$, write

$$
w=W-S(t) e^{-b x}, \quad b=|\alpha|+1>0, \quad S(t)=\frac{\Delta R(t)}{\alpha-b} .
$$

One has $W \in H^{2}[0, \infty)$ and $W_{x}=w_{x}+b S(t) e^{-b x}$. Thus

$$
\begin{aligned}
W_{x}(0, t)+\alpha W(0, t) & =w_{x}(0, t)+b S(t)+\alpha(w(0, t)-S(t)) \\
& =\Delta P(t)+b S(t)+\alpha \Delta Q(t)-\alpha S(t) \\
& =\Delta R(t)+(b-\alpha) S(t) \\
& =0
\end{aligned}
$$

and (2.4) becomes

$$
W_{t}=-i W_{x x}-i k|W|^{2} W+G_{0}+G_{1}+G_{2} .
$$

It might be cumbersome to write exactly what those $G_{0}, G_{1}, G_{2}$ are, but for now we only need to indicate that

$$
\begin{aligned}
\left\|G_{0}\right\|_{2} & \leq c_{1}\|R\|_{C^{1}[0 . T]}, \\
\left\|G_{j}(0)\right\|_{2} & \leq c_{2}\left\|W_{0}\right\|_{2}, \quad j=1,2,
\end{aligned}
$$

and

$$
\begin{aligned}
& \left\|G_{0}^{\prime}(t)\right\|_{2} \leq c_{3}\|\Delta R\|_{C^{2}[0 . T]}, \\
& \left\|G_{j}^{\prime}(t)\right\|_{2} \leq c_{4}\left(\|W\|_{2}+\left\|W_{t}\right\|_{2}\right), \quad j=1,2 .
\end{aligned}
$$

From $W_{0}=W(x, 0)=w_{0}(x)-S(0) e^{-b x}$ we see that $W_{0}(0)=w_{0}(0)-S(0)=0$. Generally $G_{1}, G_{2} \in D(A)$ but $G_{0} \notin D(A)$. But since $S \in C^{2}, G_{0}=G_{0}\left(e^{-b x}, S, S^{\prime}\right)$, $G_{0}^{\prime}(t)$ is continuous. By $[3,8]$, one has

$$
\int_{0}^{t} N(t-s) G_{0}(s) d s \in D(A)
$$

where

$$
A=-i D_{x}^{2}+i a, \quad a>\frac{\alpha^{2} c^{4}}{2}
$$


with $D(A)=\left\{W, W_{x x} \in L^{2}[0, \infty) ; W(0)=0\right\}$ and $N(t)=\exp \{A t\}$ being a strongly continuous contraction semigroup in $L^{2}$. Here $c$ denotes the coefficient in the Gagliardo-Nirenberg estimate $\|u\|_{\infty} \leq c\left\|u^{\prime}\right\|_{2}\|u\|_{2}$. One then converts (2.16) into an integral equation

$$
\begin{aligned}
W(t) & =N(t) W_{0}+\int_{0}^{t} N(t-s) G_{0} d s+\int_{0}^{t}\left(G_{1}+G_{2}-i k|W|^{2} W\right) d s \\
& =N(t) W_{0}+\int_{0}^{t} N(t-s) G(s) d s \\
& =N(t) W_{0}+\int_{0}^{t} N(s) G(t-s) d s .
\end{aligned}
$$

Now $\|w\|_{1.2}$ along with $\|w\|_{\infty}$ are bounded, because by Lemma 1 one has

$$
\begin{aligned}
\|w\|_{1,2} & \leq\|u\|_{1,2}+\|v\|_{1,2} \leq 2 \tilde{\lambda}, \\
\|w\|_{\infty} & \leq\|u\|_{\infty}+\|v\|_{\infty} \leq 2 \lambda_{0},
\end{aligned}
$$

and hence $\|w\|_{1,2}$ and $\|W\|_{\infty}$ are bounded as well. By using Gagliardo-Nirenberg estimates, one obtains

$$
\left\|k|W|^{2} W\right\|_{2,2} \leq c_{0}\|W\|_{\infty}^{2}\|W\|_{2,2} \leq \bar{c}\|W\|_{2,2} .
$$

Note

$$
\|\Delta R\|_{C^{2}[0, T]} \leq\left\|R_{1}\right\|_{C^{2}[0, T]}+\|R\|_{C^{2}[0, T]} \leq 2 M .
$$

Since $W_{0} \in D(A)$, one has $\left(N(t) W_{0}\right)_{t}=N(t) A W_{0}$. By (2.23)

$$
\begin{aligned}
W_{t}(t) & =\left(N(t) W_{0}\right)_{t}+N(t) G(0)+\int_{0}^{t} N(t) G^{\prime}(t-s) d s \\
& =N(t)\left(A W_{0}\right)+N(t) G(0)+\int_{0}^{t} N(t-s) G^{\prime}(s) d s .
\end{aligned}
$$

Here

$$
G(0)=G_{0}(0)+G_{1}(0)+G_{2}(0)-i k\left|W_{0}\right|^{2} W_{0} .
$$

Since $N(t)$ is a contraction semigroup on $L^{2}$ one has

$$
\left\|N(t)\left(A W_{0}\right)\right\|_{2} \leq c_{5}\left\|W_{0}\right\|_{2,2} .
$$

Put (2.17), (2.18), and (2.26) in (2.29):

$$
\|G(0)\|_{2} \leq c_{0}\left(\|\Delta R\|_{C^{\prime}[0, T]}+\left\|W_{0}\right\|_{2,2}\right) .
$$


From (2.16)

$$
G^{\prime}(t)=G_{0}^{\prime}(t)+G_{1}^{\prime}(t)+G_{2}^{\prime}(t)-i k\left(2|W|^{2} W_{t}+W^{2} \bar{W}_{t}\right) .
$$

Put (2.19) and (2.20) in (2.32) (note $\|W\|_{\infty}$ is bounded):

$$
\left\|G^{\prime}(t)\right\|_{2} \leq c_{6}\left(\|\Delta R\|_{C^{2}[0, T]}+\|W\|_{2}+\left\|W_{t}\right\|_{2}\right) .
$$

Since $N(t)$ is a contraction semigroup, one can use (2.30), (2.33) in (2.32)

$$
\begin{aligned}
\left\|W_{t}\right\|_{2} \leq & \left\|N(t)\left(A W_{0}\right)\right\|_{2}+\|G(0)\|_{2}+\int_{0}^{t}\left\|N(t-s) G^{\prime}(s)\right\|_{2} d s \\
\leq & c_{5}\left\|W_{0}\right\|_{2,2}+c_{0}\left(\|\Delta R\|_{C^{1}[0, T]}+\left\|W_{0}\right\|_{2,2}\right) \\
& +\int_{0}^{t} c_{6}\left(\|\Delta R\|_{C^{2}[0, T]}+\|W\|_{2}+\left\|W_{t}\right\|_{2}\right) d s .
\end{aligned}
$$

By the Gronwall lemma,

$$
\begin{aligned}
\left\|W_{t}\right\|_{2} \leq & c_{5}\left\|W_{0}\right\|_{2,2}+c_{0}\left(\|\Delta R\|_{C^{1}[0, T]}+\left\|W_{0}\right\|_{2,2}\right) \\
& +c_{6}\left(T\|\Delta R\|_{C^{2}[0, T]}+\int_{0}^{t}\|W\|_{2} d s\right) \exp \left\{c_{6} T\right\} \\
\leq & c^{\prime}\left(\|\Delta R\|_{C^{2}[0, T]}+\left\|W_{0}\right\|_{2,2}\right)+\bar{c} \int_{0}^{t}\|W\|_{2} d s .
\end{aligned}
$$

Since $w=W+S(t) e^{-b x}, w_{t}=W_{t}+S^{\prime}(t) e^{-b x}$, one has

$$
\begin{aligned}
\left\|w_{t}\right\|_{2} \leq & \left\|W_{t}\right\|_{2}+\|\Delta R\|_{C^{\prime}[0, T]} \\
\leq & c^{\prime}\left(\|\Delta R\|_{C^{2}[0, T]}+\left\|w_{0}\right\|_{2,2}+c_{0}^{\prime}\|\Delta R\|_{C^{1}[0, T]}\right) \\
& +\bar{c}\left(\int_{0}^{t}\left(\|w\|_{2}+\|\Delta R\|_{C^{1}[0, T]}\right) d s\right)+\|\Delta R\|_{C^{1}[0, T]} \\
\leq & c\left(\|\Delta R\|_{C^{2}[0, T]}+\left\|w_{0}\right\|_{2,2}\right)+\bar{c} \int_{0}^{t}\|w\|_{2} d s .
\end{aligned}
$$

Now we can use Lemma 2

$$
\begin{aligned}
\|v-u\|_{Y_{2}} & =\sup _{0 \leq t \leq T}\left(\left\|w_{t}\right\|_{2}+\|w\|_{2}\right) \\
& \leq \sup _{0 \leq t \leq T}\left(c\left(\|\Delta R\|_{C^{2}[0, T]}+\left\|w_{0}\right\|_{2,2}\right)+\bar{c} \int_{0}^{t} m\|\Delta z\|_{X_{1}}^{1 / 2} d s+m\|\Delta z\|_{X_{1}}^{1 / 2}\right) \\
& \leq c\left(\|\Delta R\|_{C^{2}[0, T]}+\left\|w_{0}\right\|_{2,2}\right)+\bar{c} T m\|\Delta z\|_{X_{1}}^{1 / 2}+m\|\Delta z\|_{X_{1}}^{1 / 2} \\
& \leq \hat{c}\|\Delta z\|_{X}^{1 / 2}
\end{aligned}
$$

and Lemma 3 is proved. 
THEOREM. The map $f: X \rightarrow Y$ is continuous and thus (1.2) is well-posed.

ProOF. By Lemma 3, it suffices to show that there exists $M>0$ such that $\|v-u\|_{Y_{3}} \leq M\left\|z_{1}-z\right\|_{X}^{1 / 2}$ where $Y_{3}=C^{0}\left(H^{2},[0, T]\right)$. From (2.4)

$$
\begin{aligned}
\left\|w_{x x}\right\|_{2} & \leq\left\|w_{t}\right\|_{2}+|k|\left(\left\|w^{3}\right\|_{2}+2\left\|u w^{2}\right\|_{2}+\left\|u^{2} w\right\|_{2}+2\left\|u^{2} w\right\|_{2}+\left\|u w^{2}\right\|\right) \\
& \leq\|w\|_{Y_{2}}+|k|\left(\left\|w^{3}\right\|_{2}+3\left\|u^{2} w\right\|_{2}+3\left\|w^{2} u\right\|_{2}\right) .
\end{aligned}
$$

Put (2.13) and (2.37) in (2.38):

$$
\begin{aligned}
\left\|w_{x x}\right\| & \leq \hat{c}\|\Delta z\|_{X}^{1 / 2}+|k|\left(\|w\|_{\infty}^{2}\|w\|_{2}+3\|u\|_{\infty}^{2}\|w\|_{2}+3\|w\|_{\infty}\|u\|_{\infty}\|w\|_{2}\right) \\
& \leq \hat{c}\|\Delta z\|_{X}^{1 / 2}+|k|\left((2 c)^{2}\|w\|_{2}+3 c^{2}\|w\|_{2}+3(2 c) c\|w\|_{2}\right) \\
& =\hat{c}\|\Delta z\|_{X}^{2}+m^{\prime}\|w\|_{2} \\
& \leq \hat{c}\|\Delta z\|_{X}^{2}+m^{\prime} m\|\Delta z\|_{X_{0}}^{1 / 2} \\
& \leq \tilde{c}\|\Delta z\|_{X}^{1 / 2}
\end{aligned}
$$

By (2.13) and (2.39)

$$
\begin{aligned}
\|v-u\|_{Y_{3}} & =\sup _{0 \leq t \leq T}\left(\left\|w_{x x}\right\|_{2}+\|w\|_{2}\right) \\
& \leq \tilde{c}\|\Delta z\|_{X}^{1 / 2}+m\|\Delta z\|_{X_{0}}^{1 / 2} \\
& \leq M\|\Delta z\|_{X}^{1 / 2} .
\end{aligned}
$$

Hence (2.40) combined with Lemma 3 shows that $f: X \rightarrow Y=Y_{2} \cap Y_{3}$ is continuous at $z$. The proof of well-posedness of (1.2) is complete.

\section{Some remarks}

We wish to make a few remarks about possible blow-up situations for the forced NLS with Robin boundary data. First we give the following definition.

DEFINITION. A solution to the forced NLS (1.2) blows up at $T$ if $\sup _{[0, T]}\|u\|_{H^{1}} \rightarrow$ $\infty$ as $t \rightarrow T^{-}$.

For $T>0$ consider

$$
\begin{gathered}
i u_{t}=u_{x x}+k|u|^{2} u, \quad 0 \leq x<\infty, \quad 0 \leq t<T, \\
u(x, 0)=u_{0}(x), \quad u_{x}(0, t)+\alpha u(0, t)=R(t),
\end{gathered}
$$

where $R(t) \in C^{2}[0, T)$. The following proposition is a direct consequence of the local existence theorem in [3]. 
Proposition 1. Assume $u_{0} \in H^{2}[0, \infty), R \in C^{2}[0, T)$. Then there exists a unique classical solution for (3.1), $u \in C^{1}\left(\left[0, T_{M}\right), L^{2}\right) \cap C^{0}\left(\left[0, T_{M}\right), H^{2}\right)$, such that either $T_{M}=T$ or $T_{M}<T$ and $\|u\|_{H^{2}} \rightarrow \infty$ as $t \rightarrow T_{M}^{-}$.

Similarly, by the global existence theorem in [3], we conclude that $T_{M}=T$. We are interested in the conditions on $R$ that would trigger a blow-up at $T$. For the same problem with Dirichlet boundary data, it was suspected in [2] that a necessary and sufficient for such blow-up is $\int_{0}^{T}|Q(\tau)|^{2} d \tau=\infty$. This conjecture was proved by Guo and Wu [6].

For (3.1) we do have the following proposition.

PROPOSITION 2. Assume that $u_{0} \in H^{2}[0, \infty), R \in C^{2}[0, T)$. Then the solution to (3.1) blows up at $T$ if $\sup _{[0, t]}|R(\tau)| \rightarrow \infty$ as $t \rightarrow T^{-}$.

PROOF. If $\sup _{[0, t]}|R(t)| \rightarrow \infty$ as $t \rightarrow T^{-}$, then either sup $|Q(t)|$ or sup $|P(t)|$ must approach to $\infty$ as $t \rightarrow T^{-}$, regardless of the value of $\alpha$. Suppose $\lim _{t \rightarrow T^{-}} \sup _{[0, T]}|Q(\tau)|=\infty$. By the Gagliardo-Nirenberg estimate

$$
|Q(t)|=|u(0, t)| \leq\|u\|_{\infty} \leq c\left\|u^{\prime}\right\|_{2}^{1 / 2}\|u\|_{2}^{1 / 2}=c\|u\|_{H^{\prime}}
$$

thus $\sup _{[0, t]}\|u\|_{H^{\prime}} \rightarrow \infty$ as $t \rightarrow T^{-}$and $u$ must blow up at $T$.

On the other hand, assume that $\lim _{t \rightarrow T^{-}} \sup _{[0, t]}|P(\tau)|=\infty$. By the GagliardoNirenberg estimate again

$$
|P(t)|=\left|u_{x}(0, t)\right| \leq\left\|u_{x}\right\|_{\infty} \leq c^{\prime}\left\|u^{\prime \prime}\right\|_{2}^{3 / 4}\|u\|_{2}^{1 / 4}=c\|u\|_{H^{2}}
$$

thus $\sup _{[0, t]}\|u\|_{H^{2}} \rightarrow \infty$ as $t \rightarrow T^{-}$. In fact, by the local existence theorem in [3], this implies that $\|u\|_{H^{2}} \rightarrow \infty$ as $t \rightarrow T^{-}$. If $\|u\|_{H^{\prime}}$ remains bounded on $[0, T)$, a re-examination of the proof of the global existence theorem in [3] reveals that $\|u\|_{H^{2}}$ must be bounded as well. Thus $\|u\|_{H^{2}} \rightarrow \infty$ as $t \rightarrow T^{-}$implies that $\|u\|_{H^{1}} \rightarrow \infty$ as $t \rightarrow T^{-}$. Again, this means that the solution $u$ blows up at $T$. Either way our proposition is proved.

Evidently, $\int_{0}^{t}|R(\tau)|^{2} d \tau=\infty$ implies that $\sup _{[0, t]}|R(t)| \rightarrow \infty$ as $t \rightarrow T^{-}$, since $R \in C^{2}[0, T)$. Therefore, if $\int_{0}^{T}|R(\tau)|^{2} d \tau=\infty$ then $u$ blows up at $T$. The reverse might be true as well.

CONJECTURE. The necessary and sufficient condition for the solution to (1.3) to blow up at $T$ is $\int_{0}^{t}|R(\tau)|^{2} d \tau=\infty$. 


\section{Acknowledgments}

This research is partially supported by a Brachman Hoffman fellowship. The author wishes to thank Professor Walter Strauss for his valuable suggestions.

\section{References}

[1] M. Ablovitz and H. Segur, Solitons and inverse scattering transform (SIAM, Philadelphia, 1991).

[2] C. Bu, "On well-posedness of the forced nonlinear Schrödinger equation", Appl. Anal. 46 (1992) 219-239.

[3] C. Bu, "An initial-boundary value problem for the nonlinear Schrödinger equation", Appl. Anal. 53 (1994) 241-255.

[4] R. Carroll and C. Bu, "Solutions of the forced nonlinear Schrödinger equation (NLS) using PDE techniques", Appl. Anal. 41 (1991) 33-51.

[5] A. Fokas, “An initial-boundary value problem for the nonlinear Schrödinger equation", Physica D 35 (1989) 167-185.

[6] B. Guo and Y. Wu, "Global existence and nonexistence of a forced nonlinear Schrödinger equation", J. Math. Phys. 36 (1995) 3479-3484.

[7] L. Nirenberg, "On elliptic partial differential equations", Annali della Scuola Nor. Sup.-Pisa 13 (1959) $115-162$.

[8] A. Pazy, Semigroups of linear operators and applications to PDE (Springer, New York, 1983).

[9] V. Zakharov and A. Shabat, "Exact theory of two-dimensional self-focusing and one-dimensional self-modulation of waves in nonlinear media", Soviet Phys. -JETP 34 (1972) 62-69. 